\title{
SOME RESULTS ON LOCALLY FINITELY PRESENTABLE CATEGORIES
}

\author{
M. MAKKAI AND A. M. PITTS
}

\begin{abstract}
We prove that any full subcategory of a locally finitely presentable (l.f.p.) category having small limits and filtered colimits preserved by the inclusion functor is itself l.f.p. Here "full" may be weakened to "full with respect to isomorphisms." Further, we characterize those left exact functors $I: \mathrm{C} \rightarrow \mathrm{D}$ between small categories with finite limits for which the functor $I^{*}: \mathbf{L E X}(\mathbf{D}$, Set $) \rightarrow \operatorname{LEX}(\mathbf{C}$, Set $)$ induced by composition is full and faithful. As an application, we prove a theorem on sheaf representations, a consequence of which is that, for any site $C=(\mathbf{C}, J)$ on a category $\mathbf{C}$ with finite limits, defined by a subcanonical Grothendieck topology $J$, the closure in $\operatorname{LEX}(\mathbf{C}$, Set $)$ under small limits and filtered colimits of the models of $C$ is the whole of $\operatorname{LEX}(\mathbf{C}$, Set $)$.
\end{abstract}

Introduction. In this paper, we make contributions to the logic of 'essentially algebraic' theories. Although the terminology will be categorical, the motivation is a model-theoretical one: our interest lies in arriving at results connecting the syntax and the semantics of that logic.

The definition of 'essentially algebraic' theory can be given conveniently only in categorical terms. As is usually done, we identify such a theory with a small category having (all) finite limits. The 2-category of all such categories, with functors preserving finite limits as morphisms (1-cells), and all natural transformations as 2-cells, is denoted by Lex; LEX denotes the 2-category with the smallness restriction removed. Set, the category of (small) sets, is the 'standard' 'theory', an object of LEX. A 'model' of a theory $\mathbf{C} \in \mathbf{L e x}$ is a morphism $\mathbf{C} \rightarrow$ Set in LEX; $\operatorname{LEX}(\mathbf{C}$, Set $)$ is the 'category of models of $\mathbf{C}$ '. Talking about relations of syntax and semantics amounts, roughly, to relating $\mathbf{C}$ and $\mathbf{L E X}(\mathbf{C}$, Set). Following [KR, we could call our subject the "doctrine of Cartesian logic".

The doctrine of Cartesian logic corresponds to a logic with existential quantification restricted to cases when unique existence is assured. For a detailed account of this correspondence, cf. $[\mathrm{C}]$. The main fact of the correspondence can be stated simply. Call a first-order theory $\mathbf{T}$ over a language $\mathbf{L}$ axiomatized by sentences of the form $\forall \mathbf{x}\left(\varphi(\mathbf{x}) \rightarrow \exists^{=1} \mathbf{y} \psi(\mathbf{x}, \mathbf{y})\right)$ with $\varphi$ and $\psi$ finite conjunctions of atomic formulas $\left(\exists^{=1} \mathbf{y}\right.$ means: "there is a unique $\mathbf{y}$ such that ...") a lim-theory. Then the categories of models of lim-theories, with ordinary homomorphisms as morphisms, are the same as the categories $\operatorname{LEX}(\mathbf{C}$, Set $)$, for $\mathbf{C} \in \mathbf{L e x}$. The connection of the lim-theories themselves and objects of Lex is a bit less easy to state; it is done in $[C]$.

Received by the editors May 8, 1984 and, in revised form, April 20, 1985.

1980 Mathematics Subject Classification (1985 Revision). Primary 03G30, 18B99; Secondary 03C20, 03C52. 
The, by now, classic work [GU] gives, among others, an intrinsic characterization of categories which, up to equivalence, can be written as $\mathbf{L E X}(\mathbf{C}$, Set); such categories are called locally finitely presentable (l.f.p.). One would be tempted to call them simply algebraic categories since they seem to be comprehensive enough to include all categories of structures which are defined, in one sense or another, by 'finitary algebraic' conditions. One of the main points about l.f.p. categories is the following exactness property: $\operatorname{LEX}(\mathbf{C}$, Set) has all (small) limits and filtered colimits and they are preserved by the evaluation ('underlying-set') functors $(-) \circ C: \mathbf{L E X}(\mathbf{C}$, Set $) \rightarrow$ Set $(C \in \mathbf{C})$. In fact, l.f.p. categories are cocomplete (have colimits) as well, although the colimits are not 'standard'; they are not preserved by the evaluation functors. The mentioned facts concerning limits and filtered colimits are a consequence of a basic fact concerning Set: in Set, finite limits commute with limits and filtered colimits.

In $\S 1$, we give an essentially self-contained account of the 'duality' of small categories with finite limits and l.f.p. categories. This duality amounts to an equivalence of the 2-categories Lex ${ }^{\text {op }}$ and LFP, the 2-category of 1.f.p. categories, with morphisms the functors preserving limits and filtered colimits. This duality may be known to many people; most of it is already in [GU], and the remaining parts are more or less folklore. Some technical results used later are also put in $\S 1$.

The main part of the paper, $\S \S 2$ and 3 , was greatly inspired by Hugo Volger's paper $[\mathrm{V}]$. In a traditional model-theoretical language, Volger arrives, sometimes implicitly, at intriguing results concerning the Cartesian doctrine. One of his main results is a somewhat complicated, but very useful syntactical characterization of those theories whose class of models, considered as a full subcategory of the category of all structures of the underlying language, has limits preserved by the inclusion. One of the main results of the present paper is a purely categorical result, Proposition 2.6, which characterizes those morphisms $F: \mathbf{C} \rightarrow \mathbf{D}$ in Lex that induce a full and faithful morphism $F^{*}: \mathbf{L E X}(\mathbf{D}$, Set $) \rightarrow \mathbf{L E X}(\mathbf{C}$, Set $)$ in LFP. Although the two results concern closely related situations, their statements or their proofs look pretty unrelated.

Another result of [V] (Proposition 16) is the fact that any full subcategory of the category of all L-structures (with homomorphisms as morphisms) for which the inclusion creates limits and filtered colimits is an elementary class. Lemma 2.2 in the present paper is a more general form of this result. Using 2.2, and material from $\S 1$ we prove (Corollary 2.4) that any full subcategory of a l.f.p. category for which the inclusion creates limits and filtered colimits is again l.f.p.; and even the more general statement where the inclusion is required to be full only on isomorphisms (Proposition 2.3).

Volger's remarks after his characterization theorem (Theorem 14) seem to amount to a proof of the weaker result, Corollary 2.4 (although the result as such is not stated). However, as far as we can see, the proof is incomplete (it does not seem to follow that the categories of models of $T$ and $T^{*}$, in Volger's remarks, are equivalent).

We shall give some comments as to why 2.3 is of interest beyond its having 2.4 . as a consequence.

Finally, a result that can be deduced from the work of Volger is the fact that whenever a structure is represented as the structure of global sections of a sheaf, 
then the structure can always be constructed by using (possibly repeatedly) limits and filtered colimits on the stalks of the sheaf. The precise, and more general, statement is Theorem 3.3. Our proof of it uses two of the above-mentioned results: 2.4 and 2.6. An interesting-looking corollary concerning subcanonical Grothendieck topologies ends the paper.

1. Gabriel-Ulmer duality. Let LEX be the 2-category of all categories having finite limits; LFC the 2-category of all categories having all (small) limits and filtered colimits. The morphisms (1-cells) of LEX are all functors between categories with finite limits preserving finite limits; the 2-cells are all natural transformations between such functors; similarly for LFC. We have forgetful functors (inclusions)

$$
\begin{aligned}
& \text { LEX } \rightarrow \text { CAT, } \\
& \text { LFC } \rightarrow \text { CAT }
\end{aligned}
$$

which are faithful and 2-full: full on 2-cells.

Set, or $S$ as we will sometimes abbreviate it in this section, the category of all small sets, is an object of both LEX and LFC. Moreover, each of the LEXoperations on $S$, i.e., the finite limits, commute with each of the LFC-operations, small limits and filtered colimits, on $S$ as is well known (cf. [CWM]). We may say that Set is a symmetric (LEX, LFC)-bistructure. Now, Set as a symmetric (LEX, LFC)-bistructure gives rise, all by itself, to a pair of (2-)adjoint (2-)functors $G, F$,

$$
\text { LEX }^{\mathrm{op}} \underset{F}{\stackrel{G}{\rightleftarrows}} \mathrm{LFC}
$$

presently described.

We start with the adjunction

given by

$$
\begin{array}{cl}
\mathbf{C A T}^{\mathrm{op}} \underset{F_{0}}{\stackrel{G_{0}}{\rightleftarrows}} \mathbf{C A T} \\
\eta_{0}: \operatorname{Id}_{\mathbf{C A T}} \rightarrow G_{0} F_{0} & \text { (unit) } \\
\varepsilon_{0}: F_{0} G_{0} \rightarrow \operatorname{Id}_{\mathbf{C A T}} \mathbf{A p}^{\mathrm{op}} & \text { (counit) }
\end{array}
$$

$$
\begin{gathered}
G_{0}=\mathbf{C A T}(-, S), \quad \text { abbreviated as }(-, S), \\
F_{0}=(-, S), \\
\quad\left(\varepsilon_{0}\right)_{\mathcal{C}}: \mathcal{C} \rightarrow((\mathcal{C}, S), S): \quad \text { evaluation, } \\
\quad\left(\eta_{0}\right)_{\mathcal{A}}: \mathcal{A} \rightarrow((\mathcal{A}, \mathcal{S}), \mathcal{S}): \quad \text { evaluation. }
\end{gathered}
$$

(The fact that this is an adjunction means that the composites

$$
\begin{gathered}
G_{0} \stackrel{\eta_{0} G_{0}}{\longrightarrow} G_{0} F_{0} G_{0} \stackrel{G_{0} \varepsilon_{0}}{\longrightarrow} G_{0} \\
F_{0} \stackrel{F_{0} \eta_{0}}{\longrightarrow} F_{0} G_{0} F_{0} \stackrel{\varepsilon_{0} F_{0}}{\longrightarrow} \mathrm{F}
\end{gathered}
$$

are identities.)

The 'symmetry property' of $S$ implies that, for any $C \in \operatorname{LEX}, \operatorname{LEX}(C, S) \in$ LFC; similarly, and symmetrically, $\operatorname{LFC}(A, S) \in$ LEX for $A \in$ LFC. Also, for 
any LEX-morphism $C \stackrel{\varphi}{\rightarrow} C^{\prime}$, the functor $\operatorname{LEX}\left(C^{\prime}, S\right) \stackrel{\hat{\varphi}}{\rightarrow} \operatorname{LEX}(C, S)$ defined by composition is an LFC-morphism; of course, a dual statement can also be made. These facts give rise to the promised 2-functors

$$
\begin{aligned}
& G: \mathbf{L E X}^{\mathrm{op}} \rightarrow \mathbf{L F C}, \quad G(\mathcal{C})=\mathbf{L E X}(C, S), \\
& F: \mathbf{L F C} \rightarrow \mathbf{L E X}^{\mathrm{op}}, \quad F(\mathcal{A})=\operatorname{LFC}(\mathcal{A}, S) .
\end{aligned}
$$

To define the counit and the unit of the desired adjunction

$$
\begin{aligned}
& \varepsilon: F G \rightarrow \operatorname{Id}_{\mathbf{L E X}}{ }^{\text {op }} \\
& \eta: \operatorname{Id}_{\mathbf{L F C}}{ }^{\text {op }} \rightarrow G F
\end{aligned}
$$

we proceed as follows.

The inclusion (1) gives rise to the 'restriction' functor, for each $C \in \mathbf{L E X}$ :

$$
((C, S), S) \rightarrow(\operatorname{LEX}(C, S), S) .
$$

Pre-composing this with $\left(\varepsilon_{0}\right)_{\mathcal{c}}$, the resulting functor factors through the inclusion

$$
\operatorname{LFC}(\operatorname{LEX}(C, S), S) \rightarrow(\operatorname{LEX}(C, S), S),
$$

resulting in the functor

$$
\varepsilon_{C}: C \rightarrow \operatorname{LFC}(\operatorname{LEX}(C, S), S)
$$

This defines $\varepsilon$; the description of $\eta$ is symmetric. The fact that $\varepsilon$ and $\eta$ form an adjunction is a formal consequence of their definition together with the corresponding fact for $\varepsilon_{0}$ and $\eta_{0}$.

We are going to take full sub-2-categories (full with respect to both 1-cells and 2-cells), Lex and LFP, of LEX and LFC, respectively, so that the corresponding restriction of the above adjunction is in fact an equivalence. Lex is the full sub2-category of LEX with objects the essentially small objects of LEX (i.e., those equivalent to a small category). The following definitions describe the smallness conditions determining LFP inside LFC.

1.1 Definitions.

(i) A collection $\mathcal{G}$ of objects in a category $\mathcal{A}$ is called a collection of generators (for $A$ ) if every object in $\mathcal{A}$ can be expressed as a filtered colimit of objects in $\mathcal{G}$.

(ii) An object $A$ of a (locally small) category $A$ is called finitely presentable (f.p.) if the functor $A(A,-): \mathcal{A} \rightarrow$ Set preserves filtered colimits. $A_{\mathrm{f} . p}$. will denote the full subcategory of $A$ whose objects are finitely presentable.

(iii) A category $\mathcal{A}$ is called locally finitely presentable (l.f.p.), if $\mathcal{A} \in \mathbf{L F C}, \mathcal{A}$ is locally small and it has a small set of generators consisting of finitely presentable objects. LFP is the full sub-2-category of LFC with objects the l.f.p. categories.

REMARKS. The use of the expression "collection of generators" in (i) is slightly nonstandard. The definition of "finitely presentable" is, of course, the usual definition; in $[\mathrm{GU}]$, one finds " $\aleph_{0}$-presentable" instead. As the reader may already see, and as will be pointed out in due course, the definition of "l.f.p." given here is equivalent to that of [GU].

1.2 THEOREM (GABRIEL-UlMER DUALITY). The pair of adjoint functors (3) obtained from the symmetric (LEX, LFC)-bistructure Set restricts to an equivalence

$$
\text { Lex }^{\text {op }} \underset{\leftarrow \text { LFP. }}{\longrightarrow}
$$


In other words,

(i) if $\mathbf{C} \in \mathbf{L e x}$, then $G(\mathbf{C}) \in \mathbf{L F P}$ and $\varepsilon_{\mathbf{C}}$ is an equivalence of categories; and

(ii) if $\mathcal{A} \in \mathbf{L F P}$, then $F(A) \in \mathbf{L e x}$, and $\eta_{A}$ is an equivalence of categories.

Before the proof of the theorem, we list some facts and lemmas.

1.3. FACTS. Let $\mathbf{C} \in \mathbf{L e x}$. (i) The inclusion $\operatorname{LEX}(\mathbf{C}, S) \hookrightarrow(\mathbf{C}, S)$ creates limits and filtered colimits.

(ii) The Yoneda embedding $y: \mathbf{C}^{\mathrm{op}} \rightarrow(\mathbf{C}, S)$ is full, faithful, and sends colimits in $\mathbf{C}$ to limits in $(\mathbf{C}, \mathbf{S})$.

(iii) The Yoneda embedding $y$ is dense, i.e., for any $M \in(\mathbf{C}, S)$, the diagram $D_{M}: \mathbf{C}^{\text {op }} \downarrow M \rightarrow(\mathbf{C}, S):(C,-) \rightarrow M \mapsto(C,-)$ has colimit $M$, with the canonical colimiting cone $(C,-) \rightarrow M \mapsto(C,-) \rightarrow M$.

(iv) The diagram $D_{M}$ of (ii) is filtered if and only if $M \in \operatorname{LEX}(\mathbf{C}, S)$.

(v) $M \in \mathbf{L E X}(\mathbf{C}, S)$ is f.p. if and only if $M$ is representable, i.e., isomorphic to $(C,-)$, for some $C \in \mathbf{C}$.

These facts are, of course, well known. For completeness, we give a few words concerning their proofs. The fact that finite limits commute with limits and filtered colimits in Set implies that a limit or a filtered colimit in $(\mathbf{C}, S)$ of functors preserving finite limits is again such a functor; this shows (i). For (iii), whose proof is a straightforward computation, see [SGA4, Exp. I, 3.4, p. 19], or [CWM, Corollary X.6.3, p. 243]. For (iv), see [SGA4, Exp. I, proof of 8.3 .3 (i) $\Rightarrow($ ii), p. 77]. Since for $M \in(\mathbf{C}, S)$, and $C \in \mathbf{C},((C,-), M) \cong M(C)$ (Yoneda), the fact that each representable functor in $\operatorname{LEX}(\mathbf{C}, S)$ is f.p. follows from (i) and the fact that (filtered) colimits in $(C, S)$ are computed pointwise. Conversely, if $A$ is an f.p. object of a category $\mathcal{A}$, and $A$ is the filtered colimit colim $A_{i}$ of objects $A_{i}$, then one concludes (by considering the identity morphism $A \rightarrow \operatorname{colim}_{i} A_{i}$ ) that there is an index $i$ such that $A$ is a retract of $A_{i}$. Put $A_{i}=B$. If we have

$$
A \underset{i}{\stackrel{p}{\leftrightarrows}} B, \quad p i=\operatorname{Id}_{A},
$$

then

$$
B \underset{i p}{\stackrel{\mathrm{Id}_{B}}{\rightrightarrows}} B \stackrel{p}{\rightarrow} A
$$

is a coequalizer diagram. Thus, if $A \in A=\operatorname{LEX}(\mathbf{C}, S)$, and we use the filtered colimit representation given by (iii) and (iv) via objects coming from $\mathbf{C}$ by $y$, using (i) and the fact that $\mathbf{C}$ has finite limits we obtain that $A$ is isomorphic to a representable functor. This proves $(\mathrm{v})$.

Note that facts 1.3(i), (iii), (iv) and (v) contain the first part of the statement of 1.2(i): $G(\mathbf{C})=\mathbf{L E X}(\mathbf{C}, S) \in \mathbf{L F P}$.

The following lemma will have uses beyond Theorem 1.2.

1.4 LEMMA. Suppose that $A$ is locally small and has limits and filtered colimits which are preserved by a functor $G: A \rightarrow B$ into a locally finitely presentable category $B$. If $A$ has a generating set of objects, $\mathcal{G}$ say, then $G$ has a left adjoint $F: B \rightarrow A$.

PROOF. We have to show that for every $B \in B$, we have: $(*)_{B}$ there is an object $F B$ and a 'universal arrow' $\eta_{B}: B \rightarrow G(F B)$ 'from $B$ to $G$ ', i.e., $\eta_{B}$ such that for every arrow $B \stackrel{g}{\rightarrow} G A$, thee is a unique $f: F B \rightarrow A$ such that $g=G(f) \eta_{B}$. If we have $B=$ colim $_{i \in I} B_{i}$, a filtered colimit representation, and $(*)_{B_{i}}$ holds for all $i$, 
then $(*)_{B}$ holds as well. In fact, for each $\alpha: i \rightarrow j$ in $\operatorname{Morph}(I)$, let $F_{\alpha}$ be defined so that

$$
\begin{array}{rlc}
B_{i} & \rightarrow & G\left(F B_{i}\right) \\
\ulcorner\alpha\urcorner \downarrow & & \downarrow G F_{\alpha} \\
B_{j} & \rightarrow & G\left(F B_{j}\right)
\end{array}
$$

commute; thus, the filtered colimit $\operatorname{colim}_{i} F B_{i}$ makes sense, and we can take $F B$ to be it. Since $G$ preserves filtered colimits, we have a canonical arrow $B=\operatorname{colim} B_{i} \rightarrow$ colim $G\left(F B_{i}\right) \cong G\left(\operatorname{colim} F B_{i}\right)$; we take $\eta_{B}$ to be this arrow; it is easy to verify that it will have the required property.

By the last remark, and since $B$ is l.f.p., it suffices to show the statement $(*)_{B}$ for $B$ f.p. in $B$. Since $A$ has limits and $G$ preserves them, we can apply the Adjoint Functor Theorem "at $B$ ", i.e., we need only find a set of arrows

$$
\left\{g_{i}: B \rightarrow G\left(A_{i}\right) \mid i \in I\right\}
$$

with the property that any $g: B \rightarrow G(A)$ factors as

$$
g: B \stackrel{g_{i}}{\rightarrow} G\left(A_{i}\right) \stackrel{G(f)}{\rightarrow} G(A)
$$

for some $i \in I$ and $f: A_{i} \rightarrow A$ in $A$. But

$$
\{B \rightarrow G(A) \mid A \in \mathcal{G}\}
$$

is such a set. For, given any $A \in A$, we can express it as a filtered colimit of objects in $\mathcal{G}$, say

$$
A=\operatorname{colim}_{k \in \mathbf{K}} A_{k}
$$

with colimiting cone

$$
\left(i_{k}: A_{k} \rightarrow A \mid k \in \mathbf{K}\right) .
$$

Since $G$ preserves filtered colimits, we have $G A \cong \operatorname{colim}_{k \in \mathbf{K}} G\left(A_{k}\right)$ and

$$
\left(G\left(i_{k}\right): G\left(A_{k}\right) \rightarrow G(A) \mid k \in \mathbf{K}\right)
$$

is a colimiting cone. But now given any $g: B \rightarrow G(A)$, since $B$ is finitely presentable, $g$ factors through one of the maps in this colimiting cone, i.e., $g$ factors as

$$
g: B \rightarrow G\left(A_{k}\right) \stackrel{G\left(i_{k}\right)}{\longrightarrow} G(A)
$$

for some $k \in \mathbf{K}$; and $A_{k} \in \mathcal{G}$ as required. $\square 1.4$

1.5 Corollary. Let $A \in \mathbf{L F P}$. For $A \in A_{\text {f.p. }}$ clearly the representable functor $A(A,-) \in \mathbf{L F C}(\mathcal{A}, S)$. Hence we have a canonical functor

$$
h_{A}: \mathcal{A}_{\mathrm{f} . \mathrm{p} .}^{\mathrm{op}} \rightarrow \operatorname{LFC}(\mathcal{A}, S) \quad(A \mapsto A(A,-)) .
$$

This functor is an equivalence of categories.

PROOF. As a consequence of the Yoneda lemma, $h_{\mathcal{A}}$ s full and faithful. To show that $h_{A}$ is essentially surjective on objects, let $X: A \rightarrow S$ be a functor in LFC. By 1.4, $X$ has a left adjoint $Y$. The adjunction $Y \dashv X$ specializes to the natural isomorphisms

$$
\mathcal{A}(Y(1), A) \cong S(1, X A) \cong X A
$$

for any $A \in A$, i.e.

$$
\mathcal{A}(Y(1),-) \cong X
$$


Since $X$ preserves filtered colimits, $Y(1)$, by definition, is f.p. $\square 1.5$

Now, we can complete the proof of $1.2(\mathrm{i})$; the assertion to be proved is that $\varepsilon_{\mathbf{C}}$ is an equivalence of categories, for $\mathbf{C} \in \mathbf{L e x}$.

Let $A=\operatorname{LEX}(\mathbf{C}, S)$. $A$ is l.f.p. By $1.3(\mathrm{v})$, the Yoneda embedding $\mathbf{C}^{\text {op }} \rightarrow A$ factors through $\mathcal{A}_{\text {f.p. }} \hookrightarrow$ incl. $A$, and in fact, the resulting functor $\mathbf{C}^{\text {op }} \rightarrow \mathcal{A}_{\text {f.p. }}$ is an equivalence of categories. Moreover, the composite

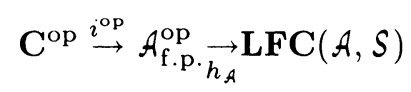

(with $h_{\mathcal{A}}$ from 1.5) is isomorphic to $\varepsilon_{\mathbf{C}}$. Therefore, by $1.5, \varepsilon_{\mathbf{C}}$ is an equivalence as well. $\square 1.2(\mathrm{i})$

Turning to the proof of 1.2 (ii), we let $\mathcal{A}$ be an l.f.p. category, $\mathbf{C}=\mathcal{A}_{\text {f.p. }}^{\text {op }}, \mathbf{B}=$ $\operatorname{LFC}(\mathcal{A}, S), i: A_{\text {f.p. }} \hookrightarrow A$ the inclusion, $h: \mathbf{C} \rightarrow \mathbf{B}$ the functor of 1.5 . By $1.5, h$ is an equivalence of categories.

It is clear that $\operatorname{LFC}(\mathcal{A}, S)$ has finite limits preserved by the inclusion

$$
\operatorname{LFC}(A, S) \rightarrow(A, S) .
$$

It follows from $h_{\AA}$ being an equivalence that $A_{\text {f.p. }}$ has finite colimits preserved by

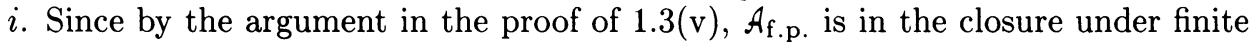
colimits of a small set contained in $\AA_{\text {f.p. }}, A_{\text {f.p. }}$ is essentially small. It follows that $F(\mathcal{A})=\mathbf{B} \simeq \mathbf{C} \in \mathbf{L e x}$, as required for the first part of 1.2(ii).

$h$ induces the equivalence $h^{*}: \mathbf{L E X}(\mathbf{B}, \mathcal{S}) \rightarrow \operatorname{LEX}(\mathbf{C}, \mathcal{S})$. Composing $\eta=$ $\eta_{\mathcal{A}}: A \rightarrow \operatorname{LEX}(\mathbf{B}, S)$ and $h^{*}$, we obtain a functor

$$
\tau: \mathcal{A} \rightarrow \operatorname{LEX}(\mathbf{C}, S)
$$

which, as inspection shows, is identical to

$$
A \mapsto A(i(-), A) .
$$

Since $h^{*}$ is an equivalence, to show that $\eta$ is an equivalence, it suffices to show that $\tau$ is one.

The fact that the functor $\tau$ is an equivalence, for any l.f.p. category $\mathcal{A}$, with a suitable definition of "l.f.p.", is due to Gabriel and Ulmer, and is arguably the main result of [GU]. We are going, nevertheless, to outline the proof, especially since some ingredients of it will be useful for other purposes as well. First, we give a concept which will be important to us. The lemma that follows the definition is due to [GU]; the second part of it is relatively little known.

1.6 Definition. A collection $C$ of objects in a category $A$ will be called conservative if, given $f: A \rightarrow B$ in $A$ such that for each $C \in C$

$$
f_{*}: \mathcal{A}(C, A) \rightarrow \mathcal{A}(C, B)
$$

is a bijection, then $f$ is an isomorphism. Thus, assuming $A$ to be locally small, $C$ is conservative iff the restricted Yoneda functor, $A \rightarrow \operatorname{Set}^{C^{\circ P}}$ (sending $A \mapsto A(-, A)$ ) reflects isomorphisms.

1.7 Lemma. Let $A$ be a locally small category with filtered colimits.

(i) Any set of generators in $A$ is a conservative set. 
(ii) If $C$ is a conservative set of finitely presentable objects in $A$ which when regarded as a full subcategory of $A$ has finite colimits that are preserved by the inclusion $i: C \hookrightarrow A$, then $i$ is dense.

ProOF. (i) This is an easy exercise in category theory.

(ii) (Cf. 7.4 of $[\mathrm{GU}]$.) To say that $i$ is dense is to say that, given an object $A \in A$, the forgetful functor $U_{A}: C / A \rightarrow A$ from the category of objects of $C$ over $A$ has

$$
\left(f: U_{A}(f) \rightarrow A \mid f \in C / A\right)
$$

as a colimiting cone. Note that by assumption on $C, C / A$ is small and filtered; hence we can form $\operatorname{colim}_{C / A} U_{A}$ in $A$. Let

$$
\left(i_{f}: U_{A}(f) \rightarrow \operatorname{colim}_{h \in C / A} U_{A}(h) \mid f \in C / A\right)
$$

be the colimiting cone and let

$$
g: \operatorname{colim}_{C / A} U_{A} \rightarrow A
$$

be the unique morphism making

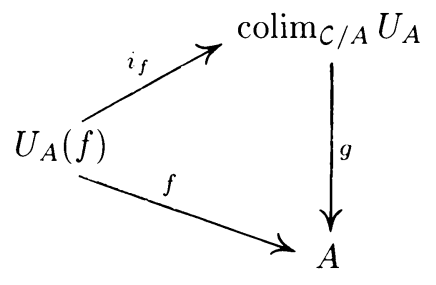

commute for each $f \in C / A$. We have to show that $g$ is an isomorphism and since $C$ is a conservative set of objects, it suffices to show that for each $C \in C$

$$
g_{*}: A\left(C, \operatorname{colim}_{f \in C / A} U_{A}(f)\right) \rightarrow A(C, A)
$$

is a bijection. But $C$ is finitely presentable; so it suffices to show that the composition with the canonical bijection

$$
G: \operatorname{colim}_{f \in C / A} A\left(C, U_{A}(f)\right) \stackrel{\cong}{\rightarrow} A\left(C, \operatorname{colim}_{C / A} U_{A}\right) \stackrel{g^{*}}{\rightarrow} A(C, A)
$$

is a bijection.

Now, this canonical bijection is that induced by the maps $\left(i_{f}\right)_{*}: A\left(C, U_{A}(f)\right) \rightarrow$ $A\left(C\right.$, colim $\left._{C / A} U_{A}\right)$. Recalling that we may form the colimit of a filtered diagram of sets as the quotient of the disjoint union of the sets by a suitable equivalence relation, we see that if $[h] \in \operatorname{colim}_{C / A} A\left(C, U_{A}\right)$ denotes the equivalence class represented by $h: C \rightarrow U_{A}(f)$, then

$$
G[h]=g_{*}\left(i_{f}\right)_{*} h=g i_{f} h=f h .
$$

But since $\left[C \stackrel{h}{\rightarrow} U_{A}(f)\right]=\left[C \stackrel{\mathrm{id}_{C}}{\longrightarrow} U_{A}(f h)\right]$ in $\operatorname{colim}_{C / A} A\left(C, U_{A}\right)$, it follows that $G$ is indeed a bijection with inverse the map sending $k: C \rightarrow A$ to $\left[\operatorname{id}_{c}: C \rightarrow U_{A}(k)\right]$. 
1.8 COROllary. If $A$ is l.f.p., then the inclusion $i: \mathcal{A}_{\text {f.p. }} \hookrightarrow A$ is dense.

Proof. By defintion of "l.f.p.", and 1.7(i), $\mathcal{A}_{\text {f.p. }}$ is a conservative set in $\mathcal{A}$. We said above that $\mathcal{A}_{\text {f.p. }}$. has finite colimits preserved by $i$. Thus, the assertion follows from 1.7(ii). $\quad 1.8$

We return to the proof of 1.2(ii), specifically of the fact that the functor $\tau$ is an equivalence. By (the dual of) [CWM, X.6.2, p. 242] and 1.8, $\tau$ is full and faithful (indeed, this fact is equivalent to the density of $i$ ). To show that $\tau$ is essentially surjective, let $M \in \mathbf{L E X}\left(\mathcal{A}_{\mathrm{f} . \mathrm{p} \text {. }}, S\right)$ be arbitrary; $M$ can be represented (1.3(iii) and (iv)) as a filtered colimit $M \cong \operatorname{colim}_{k} A_{\text {f.p. }}^{\text {op }}\left(A_{k},-\right) \cong \operatorname{colim}_{k} A\left(i(-), A_{k}\right)$; but the

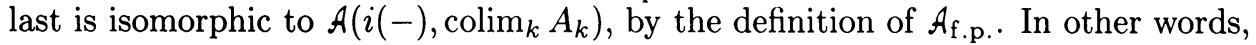
$M \cong \tau(A)$ for $A=\operatorname{colim}_{k} A_{k}$, as was to be shown. $\square 1.2(\mathrm{ii})$

The following characterization of l.f.p. categories appears in $[\mathrm{Mu}]$ (the referee's communication).

1.9 COROLlaRY. The locally small category $A$ is l.f.p. if and only if it satisfies the following conditions

(i) A has filtered colimits.

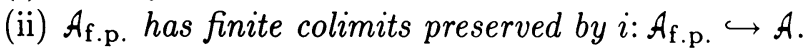

(iii) A has a small set of generators consisting of f.p. objects.

PROOF. It is clear from the above that every l.f.p. category $A$ satisfies condition (ii). Conversely, one sees that the conclusion of 1.8 remains true for $A$ satisfying the three conditions, with the same proof. Thus, the final argument above establishes that the canonical functor $\tau: \mathcal{A} \rightarrow \operatorname{LEX}\left(\mathcal{A}_{\mathrm{f} . \mathrm{p} .}^{\mathrm{op}}, S\right)$ is an equivalence in this case; also, $\mathcal{A}_{\mathrm{f} . \mathrm{p} .}^{\text {op }}$ is essentially small. $\quad \square 1.9$

The official definition of "l.f.p." in [GU] is this: $A$ is l.f.p. iff $A$ is co-complete (has all small colimits), and it has a small conservative set contained in $\mathcal{A}_{\text {f.p. }}$. With out definition of "l.f.p.", this is a true statement: the 'if' part is contained in 1.9 and 1.7(ii); the 'only if' part follows by the fact that $\operatorname{LEX}\left(\mathcal{A}_{\mathrm{f} . \mathrm{p} .}^{\mathrm{op}}, S\right)$ is a full and reflective subcategory of $\left(A_{\text {f.p. }}^{\text {op }}, S\right)$ (a consequence of 1.4$)$, which implies that $\operatorname{LEX}\left(\mathcal{A}_{\mathrm{f} . \mathrm{p} .}^{\mathrm{op}}, S\right)$ is co-complete [CWM, Exercise V.5.3, p. 116].

Here is a new set of conditions ensuring that a category is l.f.p.

1.10 Proposition. Suppose $B$ is l.f.p., A has limits and filtered colimits, $G: A \rightarrow B$ preserves them, and $G$ is conservative (reflects isomorphisms). Suppose either that (i) $A$ has a small set of generators or that (ii) $G$ has a left adjoint and $\mathcal{A}_{\text {f.p. }}$ has finite colimits preserved by the inclusion $\mathcal{A}_{\text {f.p. }} \hookrightarrow A$. Then $A$ is l.f.p. Moreover, $A_{\text {f.p. }}$ is equal to the finite colimit closure of $\left\{F(B): B \in B_{\text {f.p. }}\right\}$, for $F$ the left adjoint of $G$.

For the proof, we need

1.11 Lemma. Suppose $A$ is locally small, has limits, and has a small set of

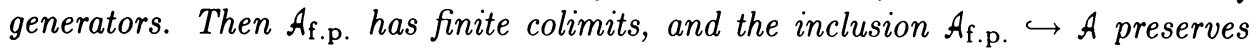
them.

PROOF. We have to show that given a finite category $\mathbf{J}$, every diagram $D: \mathbf{J} \rightarrow \mathcal{A}$ whose vertices are finitely presentable has a colimit in $A$ (since this colimit will automatically be finitely presentable). In other words, given such a diagram we 
have to find a cone $\left(a_{j}: D(j) \rightarrow A \mid j \in \mathbf{J}\right)$ under $D$ which is initial amongst such cones; but since $A$ has limits, it is sufficient to verify a "solution set" condition (cf. Theorem V.6.1 of [CWM]), i.e., find a set of cones under $D$ so that an arbitrary cone under $D$ factors through one of the cones in the set. We claim that if $C$ is a set of generators for $\mathcal{A}$, then the collection of cones under $D$ with vertices in $\mathcal{G}$ forms such a solution set. (It is a set since $\mathbf{J}$ and $\mathcal{G}$ are small and $\mathcal{A}$ is locally small.) For suppose $\left(a_{j}: D(j) \rightarrow A \mid j \in \mathbf{J}\right)$ is an arbitrary cone under $D$. Express $A$ as a filtered colimit of objects in $\mathcal{G}$, say

$$
A=\operatorname{colim}_{k \in \mathbf{K}} G(k)
$$

with colimiting cone

$$
\left(i_{k}: G(k) \rightarrow A \mid k \in \mathbf{K}\right) .
$$

Since each $D(j)$ is finitely presentable, $a_{j}$ factors through one of the $i_{k}$ :

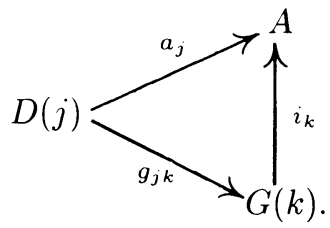

Since there are only finitely many objects $j \in \mathbf{J}$ and $\mathbf{K}$ is filtered, we can take the same $k$ for every $j$ in the above; then since there are only finitely many arrows $\alpha: j \rightarrow j^{\prime}$ in $\mathbf{J}$, by changing $k$ if necessary we can also arrange (by using now the 'uniqueness condition' in the $D(j)$ 's being finitely presented) that for each $\alpha$

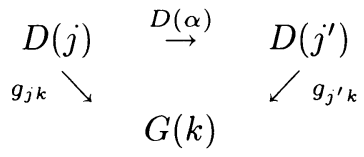

commutes. Thus $\left(g_{j k}: D(j) \rightarrow G(k) \mid j \in \mathbf{J}\right)$ is a cone in the solution set through which the given cone factors (via $i_{k}$ ). $\square 1.11$

PROOF OF 1.10. Assume the hypotheses of the proposition and condition (i), in

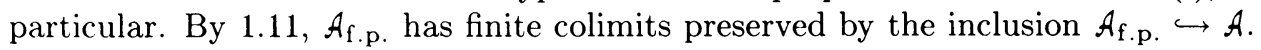
By $1.4, G$ has a left adjoint, say $F$. In other words, condition (ii) is satisfied too.

Consider the objects of $A$ of the form $F B$, for $B \in B_{\mathrm{f} . p .}$. Using the adjunction and the fact that $G$ preserves filtered colimits, one immediately checks that $F B \in A_{\mathrm{f} \text {.p. }}$ when $B \in B_{\text {f.p. }}$. We claim that the set $\left\{F B: B \in B_{\text {f.p. }}\right\}$ is conservative for $A$. In fact, with any $f: A \rightarrow A^{\prime}$ in $A$, and $B \in B_{\text {f.p. }}$, the map in Set

$$
(F B, A) \underset{(F B, f)}{\longrightarrow}\left(F B, A^{\prime}\right)
$$

is isomorphic to

$$
(B, G A) \underset{(B, G f)}{\rightarrow}\left(B, G A^{\prime}\right)
$$

by the adjunction $F \dashv G$. Therefore, if the former is a bijection, so is the latter. Since $B_{\text {f.p. }}$ is conservative in $B(1.7(\mathrm{i}))$, it follows that if the former maps are all bijections, $G f$ is an isomorphism; hence, since $G$ reflects isomorphisms, $f$ is an isomorphism as well. This shows the claim. 
Take the closure of $\left\{F B: B \in B_{\text {f.p. }}\right\}$ under finite colimits; call this closure $C$. $C$ is an essentially small full subcategory of $A_{\text {f.p. }}$ having finite colimits preserved by the inclusion $C \hookrightarrow A$. By 1.7 (ii), $C$ is dense in $A$. It follows that $A$ is l.f.p., and $C=A_{\text {f.p. }}$ (the latter by the argument given for $1.3(\mathrm{v})$ ). $\quad \square 1.10$

Finally, let us note a consequence of 1.4 , and introduce a piece of notation.

1.12 Corollary/Definition. Every morphism $F: A \rightarrow B$ in LFP has a left adjoint. We write $F$ ! for the essentially unique left adjoint of $F$.

Let us note that all results in this section remain valid, with essentially the same proofs, upon systematically replacing, in all definitions and assertions, the term "finite" by the term "of cardinality less than $\kappa$ " for a fixed regular cardinal $\kappa$. In fact, this is the context in which the results of $[\mathrm{GU}]$ are stated.

2. Reflective subcategories of l.f.p. categories. Throughout this section we will consider a fixed locally finitely presentable category $B$, a category $A$ with limits and filtered colimits and a functor $F: A \rightarrow B$ preserving these limits and colimits.

By Theorem 1.2 we can take $B$ to be $\operatorname{LEX}(\mathbf{C}$, Set $)$ with $\mathbf{C}$ a small category with finite limits. Thus if $\mathbf{L}$ is the underlying graph of $\mathbf{C}$, objects of $B$ can be regarded as certain kinds of diagram of type $\mathbf{L}$ in Set, the category of all such diagrams being denoted $\operatorname{Set}^{\mathbf{L}}$. Now we can regard $\mathbf{L}$ as specifying a many-sorted language: the objects of $\mathbf{L}$ are the sorts and the arrows of $\mathbf{L}$ the (unary) function symbols. From this viewpoint, diagrams of type $\mathbf{L}$ in Set are just $\mathbf{L}$-structures in the usual sense of model theory. In prticular the essential image of $F$, viz

$$
\mathcal{K}=\{B \in B \mid B \cong F(A) \text { some } A \in A\},
$$

is a class of L-structures and we can apply the terminology and methods of model theory to study it. We thus have the notion of an elementary embedding $M \rightarrow N$ (a particular kind of morphism in $\operatorname{Set}^{\mathbf{L}}$ ), the concept of two objects $M, N \in \operatorname{Set}^{\mathbf{L}}$ being elementarily equivalent (denoted $M \equiv N$ ), ultraproducts of objects of Set $^{\mathbf{L}}$, etc. We also need the following model-theoretic concepts:

An ultralimit of an $\mathbf{L}$-structure $M$ is a filtered colimit of the form $\operatorname{colim}_{\beta<\alpha} M_{\beta}$, where $\alpha$ is an ordinal, $M_{0}=M$ and for each $\beta$ with $\beta+1<\alpha, M_{\beta} \rightarrow M_{\beta+1}$ is the diagonal embedding of $M_{\beta}$ into an ultrapower of itself; moreover there is a "continuity" condition, namely that for each limit ordinal $\lambda<\alpha, M_{\lambda}=\operatorname{colim}_{\beta<\lambda} M_{\beta}$.

It is well-known that ultraproducts in Set $^{\mathbf{L}}$ are combinations of products and filtered colimits; by their definition ultralimits are such combinations too. More precisely, if $A$ is a (not necessarily full) subcategory of $\operatorname{Set}^{\mathbf{L}}$, with the inclusion $A \hookrightarrow$ Set $^{\mathrm{L}}$ creating all limits and filtered colimits, and $M \in A$, then all ultralimits of $M$ are (up to isomorphism) in $A$ as well.

Now let $\kappa$ be an infinite cardinal and $M$ an L-structure. Say that $M$ is $\leq \kappa$ saturated if for all sets $I$ of cardinality $\leq \kappa$, for any $I$-indexed system $\left(a_{i} \mid i \in I\right)$ of elements of $M$ and for any set $\Phi(x)$ of formulae with single free variable $x$ in the language $\mathbf{L}$ extended by adding the $a_{i}$ as constants, if every finite subset of $\Phi(x)$ is satisfiable in $M$, then so is $\Phi(x) . M$ is called $\leq \kappa$-homogeneous if for any sets $I, J$ of cardinality $\leq \kappa$, any map $f: I \rightarrow J$ and any indexed systems $\left(a_{i} \mid i \in I\right)$, $\left(b_{j} \mid j \in J\right)$ of elements of $M$, if

$$
\left(M,\left(a_{i} \mid i \in I\right)\right) \equiv\left(M,\left(b_{f(i)} \mid i \in I\right)\right)
$$


then there is an automorphism $h$ of $M$ with $h\left(a_{i}\right)=b_{f(i)}$, all $i \in I$.

2.1 LEMMA. For $\kappa$ and $M$ as above, there is an ultralimit of $M$ which is $\leq \kappa$ saturated and $\leq \kappa$-homogeneous.

PROOF. The result follows from Theorems 6.1.8, 6.1.4 and 5.1.17 of [CK]. $\square 2.1$

2.2 LEMMA. With $F: A \rightarrow B, \mathbf{L}$ and $K$ as above, suppose that $F$ is faithful and full on isomorphisms, i.e., if $g: F A \rightarrow F A^{\prime}$ is an isomorphism in $B$, then $g=F(f)$ for some (isomorphism) $f: A \rightarrow A^{\prime}$ in $A$. Then

(i) $\mathcal{K}$ is an elementary class of $\mathbf{L}$-structures, i.e., there is a first-order theory $\mathbf{T}$ in the language $\mathbf{L}$ with $\mathrm{K}=\operatorname{Mod}(\mathbf{T})$ the class of models of $\mathbf{T}$ in Set.

(ii) $F$ is full for elementary embeddings, i.e., if $g: F A \rightarrow F A^{\prime}$ is an elementary embedding of $\mathbf{L}$-structures, then $g=F(f)$ for some $f: A \rightarrow A^{\prime}$ in $A$.

ProOF. (i) By assumption on $F$ its essential image $K=\{B \mid B \cong F A$ some $A \in A\}$ is closed under taking ultraproducts and ultralimits. However it is also closed under elementary equivalence. For suppose $M \equiv N$ with $N \in \mathcal{K}$. Taking $\kappa \geq \operatorname{card} M\left(=\sum\{\operatorname{card} M(c) \mid c \in \mathbf{L}\}\right)$, by Lemma 2.1 there is a $\leq \kappa$-saturated, $\leq \kappa$-homogeneous $\mathbf{L}$-structure $P$ which is an ultralimit of $N$ and hence in $K$. By saturation there is an elementary embedding $e: M \rightarrow P$ (cf. 5.1 .12 of $[\mathrm{CK}])$. We will show that $e$ is the joint equalizer of the automorphisms $h$ of $P$ with $h e=e$ : it then follows by assumption on $F$ (in particular since $F$ is full on isomorphisms) that $M \in K$.

So given $a \in P \backslash \operatorname{im}(e)$ we have to find an automorphism $h$ of $P$ such that $h e=e$ but $h(a) \neq a$. By the homogeneity of $P$ it suffices to find $b \in M$ with

$$
(P, a,(e(c) \mid c \in M)) \equiv(P, b,(e(c) \mid c \in M))
$$

and $b \neq a$. Such $b$ satisfies the formulae in the set

$$
\Phi(x)=\{a \neq x\} \cup\{\varphi(x, e(\mathbf{c})) \mid \mathbf{c} \in M \text { and } P \vDash \varphi(a, e(\mathbf{c}))\} .
$$

By saturation, we can find $b$ provided each finite subset of $\Phi(x)$ is satisfied in $P$. But if

$$
a \neq x \wedge \psi(x, e(\mathbf{c}))
$$

is the conjunction of such a finite set which is not satisfied in $P$, then

$$
P \vDash \forall x(\psi(x, e(\mathbf{c})) \rightarrow x=a) .
$$

Now $x=a$ shows that

$$
P \vDash \exists x \psi(x, e(\mathbf{c}))
$$

and $e$ is elementary: so there is $c_{0} \in M$ with

$$
P \vDash \psi\left(e\left(c_{0}\right), e(\mathbf{c})\right) .
$$

Hence $a=e\left(c_{0}\right)$, contradicting $a \notin \operatorname{im}(e)$.

Thus $K$ is closed under ultraproducts and elementary equivalence and hence it is an elementary class (cf. [CK]).

(ii) Given the elementary embedding $g: F A \rightarrow F A^{\prime}=N$, find $e: N \rightarrow P$ as in the proof of (i). Thus $g$ and $e g$ are the equalizers of collections of automorphisms 
of $P$ and hence (changing $P$ by an isomorphism if necessary) are in the image of $F$. But since

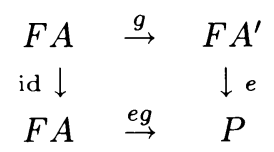

is a pullback square, it follows that $g$ is also in the image of $F$. $\square 2.2$

REMARK. Lemma 2.2 for the case when $F$ is full is due to Volger; cf. Proposition 16 in $[\mathrm{V}]$.

2.3 Proposition. Given a functor $F: A \rightarrow B$ with $B \in \mathbf{L F P}$ and such that $A$ has and $F$ preserves limits and filtered colimits, suppose that $F$ is faithful and full on isomorphisms. Then $A \in \mathbf{L F P}$ (and hence $F$ is a morphism in LFP).

PROOF. By the Löwenheim-Skolem theorem, every L-structure is the colimit of a directed diagram of $\mathbf{L}$-structures of power at most card $\mathbf{L}+\aleph_{0}$ and of elementary embeddings. Hence, by $2.2, A$ has an essentially small set of generators, consisting of those objects that are, as $\mathbf{L}$-structures, of power at most card $\mathbf{L}+\aleph_{0}$. Now, the result follows from 1.10 . $\square 2.3$

REMARKS. 1. The referee suggested that the above proof could be made more categorical by employing the methods and results of [AN1, AN2, and NS], or the equivalent versions given by [GL].

2. To put ourselves in a familiar situation, let $B$ the category of $\mathbf{L}$-structures, for an arbitrary fixed language $\mathbf{L}$. Let $K$ be an arbitrary class of $\mathbf{L}$-structures (objects in $B$ ). Then there is, up to equivalence, a smallest subcategory (not necessarily full) $[K]_{s}$ of $B$ containing $K$ such that the inclusion $[K]_{s} \rightarrow B$ is full on isomorphisms, creates limits and filtered colimits. $[K]_{s}$ is constructed by starting with objects in $K$ and by successively throwing in the objects and morphisms of limiting and filtered colimiting cones based on diagrams already in, as well as all isos between objects already in $[K]_{s}$. The structures in $[K]_{s}$ can be said to share the common algebraic properties of the ones in $K$, in a rather wide sense of 'algebraic', since algebraic properties should be preserved under the operations that are used to build $[K]_{s}$ from $K$. Eg., let $L$ contain the single relation symbol $\subset$, and let $K$ be the class of all partial orders $O(X)$ of open sets of any topological space $X$. Then the properties summarized in " $M$ is a Heyting algebra" will all be shared by the structures $M$ in $[K]_{s}$. Note that the quoted properties are expressed in terms of $\subset$ by first-order sentences of fairly high complexity; nevertheless, they are to be regarded as 'algebraic', in contrast to arbitrary first-order properties.

Lemma 2.2 says that the class of objects in $[K]_{s}$ is an elementary class; in other words that, conversely, any structure that shares all those first-order properties common to members of $[K]_{s}$ is, in fact, a member of $[K]_{s}$. Although we have not found a neat syntactic description of the algebraic properties, in the wide sense, common to members of $K$, we have found a simple description of the class of all structures sharing these properties. We are encouraged to define the algebraic properties of $K$ as the axioms true in $[K]_{s}$.

Proposition 2.3 reassures us that, at least abstractly, $[K]_{s}$ is an 'algebraic' category, i.e., it can be presented as $\operatorname{LEX}(\mathbf{C}$, Set) for some (although not very wellknown) $\mathbf{C} \in$ Lex. In $[\mathrm{MI}]$, further observations will be made on $\mathbf{C} \simeq\left([K]_{s}\right)_{\text {f.p. }}^{\text {op }}$. 
2.4 COROLLARY. If $A \hookrightarrow B$ is a full subcategory of a locally finitely presentable category and $A$ is closed under limits and filtered colimits in $B$, then $A$ is also locally finitely presentable and a reflective subcategory of $B . \quad \square 2.4$

If in $2.4, B=\operatorname{LEX}(\mathbf{C}$, Set), then by Lemma $2.2(\mathrm{i}), A=\operatorname{Mod}(\mathbf{T})$ for $\mathbf{T}$ some first-order theory in the language $\mathbf{L}=$ graph of $\mathbf{C}$. Volger has given a syntactic characterization of the kind of theories that arise in this way: cf. Theorem 14 of [V]. Volger's theorem says the following. Given any language $\mathbf{L}$, and an elementary class $A$ of L-structures; then $\mathcal{A}$, when considered a full subcategory of the category of L-structures, has limits and filtered colimits preserved by the inclusion if and only if $\mathcal{A}$ can be axiomatized by an $\mathbf{L}$-theory $\mathbf{T}$ of the following kind: the axioms of $\mathbf{T}$ are all of the form $\forall \mathbf{x}(\varphi(\mathbf{x}) \rightarrow \exists \mathbf{y} \psi(\mathbf{x}, \mathbf{y}))$, where $\varphi$ and $\psi$ are conjunctions of atomic formulas; moreover, for each such axiom-and, in fact, for each consequence of $T$ of the given form - there exists a formula $\sigma(\mathbf{x}, \mathbf{y}):=: \exists \mathbf{z} \tau(\mathbf{x}, \mathbf{y}, \mathbf{z})$ with $\tau$ a conjunction of atomic formulas such that

$$
T \vdash \forall \mathbf{x}(\varphi(\mathbf{x}) \rightarrow \exists \mathbf{y} \psi(\mathbf{x}, \mathbf{y}) \wedge \sigma(\mathbf{x}, \mathbf{y}))
$$

and

$$
T \vdash \forall \mathbf{x} \exists^{\leq 1} \mathbf{y} \sigma(\mathbf{x}, \mathbf{y}) .
$$

A simple way for a theory $\mathbf{T}$ to satisfy Volger's condition is to be axiomatized by sentences of the form $\forall \mathbf{x}\left(\varphi(\mathbf{x}) \rightarrow \exists^{=1} \mathbf{y} \psi(\mathbf{x}, \mathbf{y})\right)$. Volger gives an example of a theory satisfying his conditions which cannot be axiomatized in the simpler way. This complication is expressed, in the categorical context, by the following fact: it is possible that a morphsm $I: \mathbf{C} \rightarrow \mathbf{D}$ in Lex has the property that $I^{*}: \operatorname{LEX}(\mathbf{D}$, Set $) \rightarrow \operatorname{LEX}(\mathbf{C}$, Set $)$ is full and faithful, and yet, $I$ is not a quotient morphism in the appropriate sense for the 2-category Lex.

Let us say a few words about quotient morphisms in Lex. Intuitively, the morphism $I: \mathbf{C} \rightarrow \mathbf{D}$, as an interpretation of the 'theory' $\mathbf{C}$ in $\mathbf{D}$, is a quotient if $\mathbf{D}$ is obtained by adding new axioms but no new primitives to $\mathbf{C}$.

Working with categories rather than syntax, the process of adding new axioms to $\mathbf{C}$ becomes that of inverting a collection $\Sigma$ of (mono)morphisms in $\mathbf{C}$ in a way that is universal for Lex. The "category of fractions" construction $Q: \mathbf{C} \rightarrow \mathbf{C}\left[\Sigma^{-1}\right]$ (cf. [GZ]) gives an explicit way of doing this for suitable collections $\Sigma$. "Universal" here means that for any $\mathbf{D} \in \mathbf{L e x}$

$$
Q^{*}: \operatorname{Lex}\left(\mathbf{C}\left[\Sigma^{-1}\right], \mathbf{D}\right) \rightarrow \operatorname{Lex}(\mathbf{C}, \mathbf{D})
$$

is full and faithful and has essential image those functors inverting every morphism in $\Sigma$. Let us call a morphism $Q$ in Lex which arises in this way a quotient morphism. Examining the construction of $\mathbf{C}\left[\Sigma^{-1}\right]$ one can prove that $Q: \mathbf{C} \rightarrow \mathbf{D}$ in Lex is a quotient iff it satisfies the condition:

For every $g: d \rightarrow Q(c)$ in $\mathbf{D}$, there is $f: c^{\prime} \rightarrow c$ in $\mathbf{C}$ and an isomorphism $d \cong Q c^{\prime}$ in $\mathbf{D}$ so that $g$ factors as $g: d \cong Q c^{\prime} \stackrel{Q f}{\rightarrow} Q c$.

The class of quotient morphisms in Lex is orthogonal to the class of conservative morphisms, i.e., those $I: \mathbf{C} \rightarrow \mathbf{D}$ which reflect isomorphisms. These two classes form a factorization system on the 2-category Lex (cf. [FT I]); in particular $I$ is an equivalence (or more precisely full, faithful, and essentially surjective) iff it is both conservative and a quotient. 
Returning to the situation of Corollary 2.4, by Theorem 1.5 up to equivalence the inclusion $A \hookrightarrow B$ in LFP is of the form

$$
A=\operatorname{LEX}(\mathbf{D}, \text { Set }) \stackrel{I^{*}}{\rightarrow} \operatorname{LEX}(\mathbf{C}, \text { Set })=B
$$

for some $I: C \rightarrow D$ in Lex; thus $I$ has the property that $I^{*}$ is full and faithful. We can now translate the possible failure of $\mathbf{T}$ (the theory with $\operatorname{Mod}(\mathbf{T}) \simeq \mathscr{A}$ ) to be a finite limit theory quotient of $\mathbf{C}$ into the statement that $I$ need not be a quotient morphism. (Of course if $I$ is a quotient then $I^{*}$ is full and faithful.) Let us see an example of this.

2.5 EXAMPLE. Let $\mathbf{T}_{0}$ be the single-sorted theory of one constant $c_{0}$ and one binary relation $\sigma$ (written $x \sigma y$ and read " $y$ immediately succeeds $x$ ") satisfying for each $n=1,2,3, \ldots$ the axiom

$$
\left(c_{0} \sigma y_{1} \sigma \cdots \sigma y_{n} \wedge c_{0} \sigma z_{1} \sigma \cdots \sigma z_{n} \sigma z\right) \rightarrow \bigwedge_{i=1}^{n} y_{i}=z_{i}
$$

Let $\mathbf{T}_{1}$ be the extension of $\mathbf{T}_{0}$ by countably many constants $c_{1}, c_{2}, \ldots$ satisfying the additonal axiom

$$
c_{n} \sigma c_{n+1}
$$

for $n=0,1,2, \ldots$.

Thus $\mathbf{T}_{0}$ and $\mathbf{T}_{1}$ are Horn theories and the faithful forgetful functor $U: \operatorname{Mod}\left(\mathbf{T}_{1}\right)$ $\rightarrow \operatorname{Mod}\left(\mathbf{T}_{0}\right)$ is in LFP. Now axiom (i) shows that in any $\mathbf{T}_{0}$-model $\left(X, \sigma, x_{0}\right)$ there is at most one way to choose a sequence of elements in $X$ starting at $x_{0}$ and satisfying axiom (ii); i.e., each $\mathbf{T}_{0}$-model has at most one $\mathbf{T}_{\mathbf{1}}$-model structure. Moreover if $\left(X, \sigma, x_{0}, x_{1}, \ldots\right)$ is a $\mathbf{T}_{1}$-model and $f:\left(X, \sigma, x_{0}\right) \rightarrow\left(X^{\prime}, \sigma^{\prime}, x_{0}^{\prime}\right)$ is a $\mathbf{T}_{0^{-}}$ homomorphism, then $\left(X^{\prime}, \sigma^{\prime}, x_{0}^{\prime}=f x_{0}, f x_{1}, \ldots\right)$ is a $\mathbf{T}_{1}$-model. It follows that the forgetful functor $U$ is not only faithful but also full. We claim that the morphism

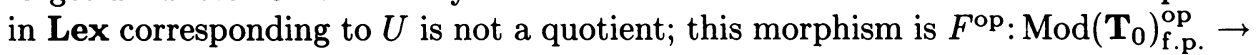
$\operatorname{Mod}\left(\mathbf{T}_{1}\right)_{\text {f.p. }}^{\text {op }}$, where $F: \operatorname{Mod}\left(\mathbf{T}_{0}\right) \rightarrow \operatorname{Mod}\left(\mathbf{T}_{1}\right)$ is left adjoint to $U$. We can describe $F$ explicitly as follows.

Given a $\mathbf{T}_{0}$-model $\left(X, \sigma, x_{0}\right)$, let $P(X)=\left\{x \mid \exists x^{\prime} x \sigma x^{\prime}\right\}$ be the subset of predecessors in $X$. By axiom (i) any (finite or infinite) sequence $x_{1}, x_{2}, \ldots$ in $P(X)$ satisfying

$$
x_{0} \sigma x_{1} \sigma x_{2} \sigma \cdots
$$

is uniquely determined. Call the longest such sequence the "spine" of $X$ : its length may be finite (possibly zero) or countably infinite. In the latter case $X$ is already a $\mathbf{T}_{1}$-model and we can take $F\left(X, \sigma, x_{0}\right)$ to be $\left(X, \sigma, x_{0}, x_{1}, \ldots\right)$ (and the unit of the adjunction at $X$ to be the identity). If however this length is finite, so that the spine is $\left(x_{1}, x_{2}, \ldots, x_{n}\right)$, we can form $F(X)$ by adjoining countably many new elements to $X$, denoted $x_{n+1}, x_{n+2}, \ldots$, and extend $\sigma$ by defining

$$
x_{n} \sigma x_{n+1} \sigma x_{n+2} \sigma \cdots \text {. }
$$

(The unit of the adjunction at $X$ will be in this case the inclusion $X \rightarrow X \cup$ $\left\{x_{n+1}, x_{n+2}, \ldots,\right\}$.)

Now the free $\mathbf{T}_{0}$-model on a set $I$ is easily seen to be $1+I$ with $\sigma$ the empty relation. $F$ thus sends this to $\mathbf{N}+I$ with

$$
\sigma=\{(n, n+1) \mid n \in \mathbf{N}\} \text {. }
$$


Let $q: F(1+1) \rightarrow F(1+0)$ be the map $\mathbf{N}+1 \rightarrow \mathbf{N}$ which sends the unique element of 1 to $1 \in \mathbf{N}$ and sends each $n \in \mathbf{N}$ to itself. It is not hard to see that a $\mathbf{T}_{0^{-}}$ model is finitely presentable iff it is finite and that the only finite $\mathbf{T}_{0}$-model $X$ with $F(X) \cong F(1)$ is 1 itself. Thus if $F^{\text {op }}: \operatorname{Mod}\left(\mathbf{T}_{0}\right)_{\mathrm{f} . \mathrm{p} \text {. }}^{\mathrm{op}} \rightarrow \operatorname{Mod}\left(\mathbf{T}_{1}\right)_{\mathrm{f} . \mathrm{p} \text {. were a quotient }}^{\text {op }}$ morphism in Lex (recalling the characterization of such morphisms given above), there would have to be $p: 1+1 \rightarrow 1$ in $\operatorname{Mod}\left(\mathbf{T}_{0}\right)$ and an automorphism $F(1) \cong F(1)$ in $\operatorname{Mod}\left(\mathbf{T}_{1}\right)$ so that $q: F(1+1) \rightarrow F(1)$ is

$$
F(1+1) \stackrel{F(p)}{\rightarrow} F(1) \cong F(1) .
$$

Of course there is just one $\mathbf{T}_{0}$-homomorphism $1+1 \rightarrow 1$, and the only automorphism of $F(1)$ is the identity: hence we would have $q=F(p)$. But these two maps differ on the unique element of $1 \hookrightarrow N+1=F(1) . \quad \square 2.5$

This situation should be contrasted with that for pretoposes (which are the proper categorical counterpart of theories in the intuitionistic logic of $=, \wedge, \vee$ and $\exists$; cf. $[\mathrm{MR}]$ and $[\mathrm{KR}])$. Specifically if $I: \mathbf{P} \rightarrow \mathbf{Q}$ is a morphism in the 2-category $\mathbf{P t}$ of (essentially small) pretoposes, the hard part of the "conceptual completeness theorem" of Reyes and the first author says that if

$$
I^{*}: \mathbf{P T}(\mathbf{Q}, \text { Set }) \rightarrow \mathbf{P T}(\mathbf{P}, \text { Set })
$$

is full and faithful then $I$ is a quotient morphism (in the sense appropriate to $\mathbf{P t}$ ); we refer the reader to Chapter 7 of $[\mathrm{MR}]$ for the details. Whilst 2.5 shows that the corresponding statement for Lex fails, one should note that nevertheless a very strong form of conceptual completeness holds for Lex by virtue of the GabrielUlmer duality; viz for $\mathbf{C}, \mathbf{D} \in \operatorname{Lex}$, if $\operatorname{LEX}(\mathbf{C}$, Set $) \simeq \mathbf{L E X}(\mathbf{D}$, Set $)$ then $\mathbf{C} \simeq \mathbf{D}$.

The natural question which therefore arises is whether one can give a condition on $I: \mathbf{C} \rightarrow \mathbf{D}$ in Lex, weaker than that of being a quotient, which is necessary and sufficient for $I^{*}: \operatorname{LEX}(\mathbf{D}$, Set $) \rightarrow \operatorname{LEX}(\mathbf{C}$, Set $)$ to be full and faithful. We devote the rest of this section to proving the following proposition, which provides a positive answer to this question.

2.6 Proposition. For $I: \mathbf{C} \rightarrow \mathbf{D}$ in $\mathbf{L e x}$, the following are equivalent:

(i) The geometric morphism $i$ Set $^{\mathbf{D}^{\mathrm{op}}} \rightarrow$ Set $^{\mathbf{C}^{\text {op }}}$ induced by $I$ (viz $i^{*}=$ left Kan extension along $I^{\mathrm{op}}$ and $i_{*}=$ precompositon with $\left.I^{\mathrm{op}}\right)$ is an inclusion.

(ii) $I^{*}: \mathbf{L E X}(\mathbf{D}$, Set $) \rightarrow \mathbf{L E X}(\mathbf{C}$, Set $)$ is full and faithful.

(iii) For each $c \in \mathbf{C}$, every object of the slice category $\mathbf{D} /$ Ic is a retract of an object in the image of the functor "Apply $I^{\prime} I^{c}: \mathbf{C} / c \rightarrow \mathbf{D} / I c$, i.e.

given $g: d \rightarrow I c$ in $\mathbf{D}$, there is $f: c^{\prime} \rightarrow c$ in $\mathbf{C}$ and maps $i: d \rightarrow I c^{\prime}$ and $r: I c^{\prime} \rightarrow d$ in $\mathbf{D}$ such that $r \circ i=\mathrm{id}, g \circ r=I f$ (and hence If $\circ i=g$ )

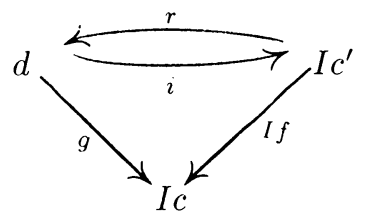


Proof. (i) $\Rightarrow$ (ii). Recall (or cf. [TT]) that if TOP denotes the 2-category of Grothendieck toposes and geometric morphisms, there is an equivalence

$$
\operatorname{LEX}(\mathrm{C}, \mathcal{E}) \simeq \operatorname{TOP}\left(\mathcal{E}, \operatorname{Set}^{\mathrm{CP}^{\mathrm{P}}}\right)
$$

that is natural in $\mathbf{C} \in \mathbf{L e x}$ and $\mathcal{E} \in \mathbf{T O P}$. In particular

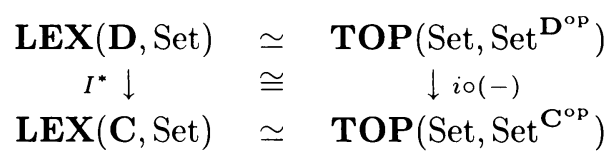

commutes up to isomorphism. But if $i$ is an inclusion, $i \circ(-)$ is full and faithful and hence so is $I^{*}$.

(ii) $\Rightarrow$ (iii). First note that if $I: \mathbf{C} \rightarrow \mathbf{D}$ is in Lex, so is $I^{c}: \mathbf{C} / c \rightarrow \mathbf{D} / I c$. Similarly, given $B$ in LFP and $B \in B$, the category $B / B$ of objects in $B$ under $B$ is locally finitely presentable. (For the forgetful functor $U: B / B \rightarrow B$ creates limits and filtered colimits, and reflects isomorphisms; it has a left adjoint $U_{!}$, given by coproduct with $B$; also, $B / B$ has arbitrary small colimits. Thus, 1.10 (with condition (ii)) is applicable.)

Moreover, given $F: A \rightarrow B$ in LFP, we get $B / F: F ! B / A \rightarrow B / B$ in LFP by sending an object under $F_{!} B, F_{!} B \rightarrow A$, to its transpose $B \rightarrow F A$ across the adjunction $F_{!} \dashv F$, and similarly for morphisms. It follows that if $F$ is full and faithful, so is $B / F$.

Now there is an equivalence of categories

$$
\mathbf{L E X}(\mathbf{C} / c, \text { Set }) \simeq Y c / \mathbf{L E X}(\mathbf{C}, \text { Set })
$$

where $Y c$ is the representable functor $\mathbf{C}(c,-)$. This is because $\Delta: \mathbf{C} \rightarrow \mathbf{C} / c$ (sending $c^{\prime}$ to $\pi_{2}: c^{\prime} \times c \rightarrow c$ ) is characterized in LEX as the result of freely adjoining a global element $1 \rightarrow c$ to $c \in \mathbf{C}$ (i.e., in terms of theories, the result of expanding the language by a constant of type $c$ without adding any new axioms). The equivalence is natural in $\mathbf{C}$ : thus given $I: \mathbf{C} \rightarrow \mathbf{D}$

$$
\begin{aligned}
& \mathbf{L E X}(\mathbf{D} / I c, \text { Set }) \simeq Y(I c) / \mathbf{L E X}(\mathbf{D}, \text { Set }) \simeq I_{!}(Y c) / \mathbf{L E X}(\mathbf{D}, \text { Set })
\end{aligned}
$$

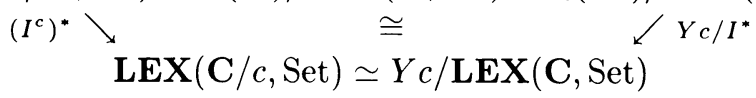

commutes up to isomorphism. (Here $I_{!}: \mathbf{L E X}(\mathbf{C}$, Set $) \rightarrow \mathbf{L E X}(\mathbf{D}$, Set) denotes the left adjoint of $I^{*}$, i.e., left Kan extension along $I$.) Thus if $I^{*}$ is full and faithful, so is $Y c / I^{*}$ and hence so is $\left(I^{c}\right)^{*}$, each $c \in \mathbf{C}$. So we will have (iii) if we can show: $I^{*}$ full and faithful implies that every object of $D$ is a retract of an object in the image of $I$. By Theorem 1.2 this is equivalent to showing that if $F: A \rightarrow B$ in LFP is full and faithful, then each $A \in \mathcal{A}_{\text {f.p. }}$ is a retract of some $F_{!} B$ with $B \in B_{\text {f.p. }}$. But given such an $F$ and $A \in \mathcal{A}_{\text {f.p. }}$, we can express $F A$ as a filtered colimit of finitely presentable objects of $B$, say

$$
F A=\operatorname{colim}_{k \in \mathbf{K}} B_{k}, \quad \text { with } B_{k} \in B_{\text {f.p. }} \text { all } k \in \mathbf{K} .
$$

Then as $F$ is full and faithful

$$
A \cong F_{!} F A \cong \operatorname{colim}_{k \in \mathbf{K}} F_{!} B_{k} .
$$


Since $A$ is finitely presentable, this composite isomorphism factors through one of the vertices of the colimit

$$
A \cdot \stackrel{\cong}{\because} \underset{k \in \mathbf{K}}{\operatorname{colim}} F_{!} B_{k}
$$

for some $k \in \mathbf{K}$. Thus $A$ is a retract of $F_{!} B_{k}$ with $B_{k} \in B_{\text {f.p. }}$, as required.

(iii) $\Rightarrow$ (i). We have to show that $i_{*}=\left(I^{\text {op }}\right)^{*}: \operatorname{Set}^{\mathbf{D}^{\text {op }}} \rightarrow \operatorname{Set}^{\mathbf{C}^{\text {op }}}$ is full and faithful. For this it is sufficient to take $X=\mathrm{Set}^{\mathrm{op}}$ in the following lemma.

2.7 Lemma. If $I: \mathbf{C} \rightarrow \mathbf{D}$ in Lex satisfies (iii) of 2.6 , then for any category $\mathcal{X}$, $I^{*}: \chi^{\mathbf{D}} \rightarrow \chi^{\mathbf{C}}$ is full and faithful.

Proof. To show $I^{*}$ is faithful, given $\varphi, \psi: F \rightrightarrows G$ in $\chi^{\mathbf{D}}$ suppose $\varphi_{I}=\psi_{I}$. Then for any $d \in \mathbf{D}$, taking $c=1$ (the terminal object in $C$ ) in 2.6(iii), we can express $d$ as a retract of an object in the image of $I$ :

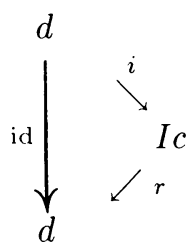

Then

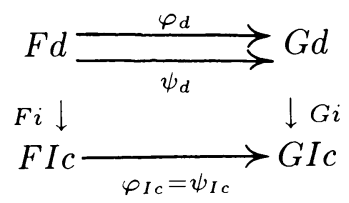

commutes and $G i$ is (split) mono: hence $\varphi_{d}=\psi_{d}$.

To show that $I^{*}$ is full, given $F, G \in \chi^{\mathbf{D}}$ suppose we have $\theta: F I \rightarrow G I$ in $\chi^{\mathbf{C}}$. Then given $d \in \mathbf{D}$ find $c, i$, and $r$ as above and define

$$
\begin{array}{lccc} 
& F d & \stackrel{\varphi_{d}}{\longrightarrow} & G d \\
\varphi_{d}=G r \circ \theta_{c} \circ F i & F i \downarrow & & \uparrow G r \\
& F I c & \overrightarrow{\theta_{c}} & G I c .
\end{array}
$$

We will show simultaneously that $\varphi_{d}$ is independent of the choice of retract and is natural in $d$.

Given $g: d_{1} \rightarrow d_{2}$ in $\mathbf{D}$ and retracts

$$
d_{\substack{\text { id } \downarrow \\ d_{j}}} \searrow_{\left\llcorner r_{j}\right.}^{i_{j}} I c_{j} \quad(j=1,2),
$$

applying $2.5($ iii $)$ to

$$
d \stackrel{\left\langle i_{1}, i_{2} g\right\rangle}{\longrightarrow} I c_{1} \times I c_{2} \cong I\left(c_{1} \times c_{2}\right)
$$


we can find $\left\langle f_{1}, f_{2}\right\rangle: c_{3} \rightarrow c_{1} \times c_{2}$ and maps $i_{3}: d_{1} \rightarrow I c_{3}, r_{3}: I c_{3} \rightarrow d_{1}$ such that

$$
r_{3} \circ i_{3}=\mathrm{id}, i_{1} \circ r_{3}=I f_{1} \quad \text { and } \quad i_{2} g \circ r_{3}=I f_{2}
$$

(and hence $I f_{1} \circ i_{3}=i_{1}$ and $I f_{2} \circ i_{3}=i_{2} g$ )

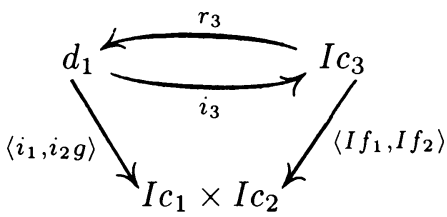

Thus

$$
\begin{aligned}
G r_{1} \theta_{c_{1}} F i_{1} & =G r_{1} \theta_{c_{1}} F I f_{1} F i_{3}=G r_{1} G I f_{1} \theta_{c_{3}} F i_{3} \\
& =G r_{1} G i_{1} G r_{3} \theta_{c_{3}} F i_{3}=G r_{3} \theta_{c_{3}} F i_{3} .
\end{aligned}
$$

Hence

$$
\begin{aligned}
G g\left(G r_{1} \theta_{c_{1}} F i_{i}\right) & =G g\left(G r_{3} \theta_{c_{3}} F i_{3}\right)=G r_{2} G i_{2} G g G r_{3} \theta_{c_{3}} F i_{3} \\
& =G r_{2} G I f_{2} \theta_{c_{2}} F i_{3}=G r_{2} \theta_{c_{2}} F I f_{2} F i_{3}=\left(G r_{2} \theta_{c_{2}} F i_{2}\right) F g .
\end{aligned}
$$

Thus $G g \circ \varphi_{d_{1}}=\varphi_{d_{2}} \circ F g$, so that $\varphi_{d}$ is natural in $d$; moreover, taking $g=$ id we also get $G r_{2} \theta_{c_{2}} F i_{2}=G r_{1} \theta_{c_{1}} F i_{1}$, i.e., the definition of $\varphi_{d}$ is independent of which retract we choose. In particular, when $d=I c$ for some $c \in \mathbf{C}$, we can take the retract $i=\mathrm{id}=r$ and conclude that $\varphi_{I c}=\theta_{c}$. Thus $I^{*}$ is indeed full. $\square 2.7$

This completes the proof of 2.6. $\square 2.6$

REMARK. The equivalence of conditions (i) and (ii) could be deduced from the "conceptual completeness result" for pretoposes, mentioned above.

3. Regaining the global sections of a sheaf from its stalks. Suppose that $\mathbf{C}$ is a small, finitely complete category and that $\mathcal{E}$ is a Grothendieck topos. We can think of a functor $F \in \operatorname{LEX}(\mathbf{C}, \mathcal{E})$ as being a "sheaf of models of $\mathbf{C}$ over the generalized space $\mathcal{E}$." From this viewpoint, the global sections of $F, \Gamma F \in$ $\operatorname{LEX}(\mathbf{C}$, Set $)$, is the composition of $F$ with $\Gamma=\mathcal{E}(1,-): \mathcal{E} \rightarrow$ Set. Similarly, given a point of $\mathcal{E}$, i.e., a geometric morphism $p \in \mathbf{T O P}(\operatorname{Set}, \mathcal{E})=\operatorname{Pt}(\mathcal{E})$, the stalk of $F$ at $p, F_{p} \in \mathbf{L E X}(\mathbf{C}$, Set $)$, is the composition of $F$ with the inverse image part of $p, p^{*}: \mathcal{E} \rightarrow$ Set. From the point of view of models of $\mathbf{C}$ in Set, $F$ provides a sheaf representation of $\Gamma F$ in terms of the stalk models $F_{p}$. Can one express this relationship solely in terms of the category of models in $\operatorname{Set}, \operatorname{LEX}(\mathbf{C}, \operatorname{Set})$ ? One often thinks of a sheaf representation as a kind of generalized limit, and indeed for presheaf toposes limits do indeed provide the answer to the above question.

3.1 EXAMPLE. Suppose that $\mathcal{E}=\operatorname{Set}^{\mathbf{A}^{\text {op }}}$ with $\mathbf{A}$ a small category. Then $\Gamma: \mathcal{E} \rightarrow$ Set is just $\lim _{\mathbf{A}}$ op , i.e., "take the limit over $\mathbf{A}_{\text {.". }}^{\text {op }}$ " Now each object $a \in \mathbf{A}$ provides a point $p_{a}$ of $\mathcal{E}$ whose inverse image part is just evaluation at $a$ :

$$
p_{a}^{*}(F)=F(a)
$$

(and similarly for morphisms in $\mathcal{E}$ ). Moreover such $\alpha: a \rightarrow a^{\prime}$ in $\mathbf{A}$ gives a natural transformation $p_{a^{\prime}}^{*} \rightarrow p_{a}$. We thus get a diagram of type $\mathbf{A}^{\text {op }}$ in $\operatorname{LEX}(\mathcal{E}, \operatorname{Set})$ whose limit is $\Gamma$ :

$$
\Gamma \cong \lim _{a \in \mathbf{A}^{\circ \mathbf{p}}} p_{a}^{*}
$$


In particular, for any $F \in \mathbf{L E X}(\mathbf{C}, \mathcal{E})$, the global sections of $F$ is expressible as a limit (in $\mathbf{L E X}(\mathbf{C}$, Set)) of stalks of $F$.

A particular case, proved by Ellerman $[\mathrm{E}]$, is when $\mathcal{E}$ is sheaves on a partially ordered set $P$ topologized by the order topology: for then $\mathcal{E} \simeq \operatorname{Set}^{\mathbf{P}^{\mathrm{op}}}$. $\square 3.1$

For general Grothendieck toposes $\mathcal{E}$, the operation of taking limits may not suffice to regain $\Gamma F$ from $\left\{F_{p} \mid p \in \mathbf{P t}(\mathcal{E})\right\}$ in $\mathbf{L E X}(\mathbf{C}$, Set $)$. Of course the latter collection of models might be rather small if $\mathcal{E}$ has few points. (Recall that it is perfectly possible for $\mathcal{E}$ to be nontrivial but have no points at all; cf. Chapter 7 of [TT].) So we will only consider toposes with enough points, i.e., such that if $f: X \rightarrow Y$ in $\mathcal{E}$ and $p^{*}(f)$ is a bijection for each $p \in \operatorname{Pt}(\mathcal{E})$, then $f$ is an isomorphism.

Now, as well as taking limits, we emphasized in $\S 1$ that the other natural operation in $\mathbf{L E X}(\mathbf{C}$, Set $)$ is that of taking filtered colimits.

3.2 Notation. If $K$ is a collection of objects in a locally finitely presentable category $A$, let $[K] \hookrightarrow A$ denote the least full subcategory of $A$ containing $K$ that is closed under taking limits and filtered colimits in $\mathcal{A}$. (We will always assume that full subcategories are replete, i.e., if an object is isomorphic to something in the full subcategory, it too is in the subcategory.) Note that by Corollary 2.4, $[K]$ is also locally finitely presentable and $[K] \hookrightarrow A$ is a morphism in LFP.

3.3 THEOREM. Suppose that $\mathbf{C}$ is a small category with finite limits, that $\mathcal{E}$ is a Grothendieck topos with enough points and that $F \in \mathbf{L E X}(\mathbf{C}, \mathcal{E})$. Then for any $L \in \mathbf{L E X}(\mathcal{E}$, Set $)$

$$
L \circ F \in\left[\left\{F_{p} \mid p \in \mathbf{P t}(\mathcal{E})\right\}\right] \hookrightarrow \mathbf{L E X}(\mathbf{C}, \text { Set }) .
$$

In particular, taking $L=\Gamma$ we get that the global sections of $F$, as a model of $\mathbf{C}$ in Set, is in the closure of the stalks of $F$ under limits and filtered colimits.

Proof. Let $\mathcal{K}=\left\{F_{p} \mid p \in \mathbf{P t}(\mathcal{E})\right\}$. Then as remarked above, by Corollary 2.4 $[K] \rightarrow \operatorname{LEX}(\mathbf{C}$, Set $)$ is in LFP, and hence by Theorem 1.2 there is $I: \mathbf{C} \rightarrow \mathbf{D}$ in Lex and an equivalence $[K] \simeq \mathbf{L E X}(\mathbf{D}$, Set $)$ making

$$
\begin{gathered}
{[K] \simeq \operatorname{LEX}(\mathbf{D}, \text { Set })} \\
\simeq \cong I^{*} \\
\mathbf{L E X}(\mathbf{C}, \text { Set })
\end{gathered}
$$

commute up to isomorphism. (Thus $\mathbf{D} \simeq[K]_{\mathrm{f} . \mathrm{p} .}^{\text {op }}$.) Hence a functor is in $[K]$ just in case it is isomorphic to one in the image of $I^{*}$.

Since $\mathcal{E}$ has enough points, we can find a sufficient set of points, i.e., a set $P$ of points such that the associated geometric morphism

$$
\pi: \operatorname{Set}^{P} \rightarrow \mathcal{E}
$$

(whose inverse image part has component at $p \in P$ equal to $\left(\pi^{*}\right)_{p}=p^{*}$ ) is a surjection. The existence of such a set $P$ is really an application of the downward Löwenheim-Skolem theorem: cf. 7.16 and 7.17 of [TT].

Now if $p \in P,\left(\pi^{*} F\right)_{p}=p^{*} F=F_{p} \in \mathcal{K} \subseteq[\mathcal{K}]$ : so by the remark above, there is $G_{p} \in \mathbf{L E X}(\mathbf{D}$, Set $)$ and an isomorphism $I^{*}\left(G_{p}\right) \simeq\left(\pi^{*} F\right)_{p}$. The functors $G_{p}$ $(p \in P)$ fit together to give a finite-limit preserving functor $G: \mathbf{D} \rightarrow \operatorname{Set}^{P}$, and by 
construction

$$
\begin{array}{rcc}
\mathbf{D} & \stackrel{G}{\rightarrow} & \operatorname{Set}^{P} \\
I \uparrow & \cong & \uparrow \pi^{*} \\
\mathbf{C} & \stackrel{F}{\rightarrow} & \mathcal{E}
\end{array}
$$

commutes up to isomorphism in LEX. We therefore get a corresponding square in TOP commuting up to isomorphism:

$\begin{array}{ccc}\operatorname{Set}^{D^{\text {op }}} & \stackrel{g}{\leftarrow} & \operatorname{Set}^{P} \\ i \downarrow & \cong & \downarrow \pi \\ \operatorname{Set}^{\mathrm{Op}} & \stackrel{f}{\leftarrow} & \mathcal{E}\end{array}$

Here $i$ is the geometric morphism induced by $I$ (as in 2.6(i)), $f$ corresponds to $F$ under the equivalence

$$
\operatorname{LEX}(\mathbf{C}, \mathcal{E}) \simeq \operatorname{TOP}\left(\mathcal{E}, \operatorname{Set}^{\mathrm{C}^{\mathrm{op}}}\right)
$$

and similarly for $g$ and $G$.

Since $I^{*}$ is full and faithful, by Proposition $2.6 i$ is an inclusion. But by construction $\pi$ is a surjection; hence the square in TOP factors as

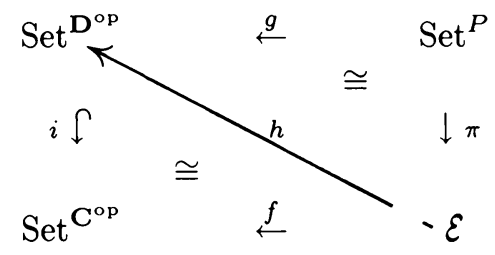

Correspondingly we thus get a factorization in LEX:

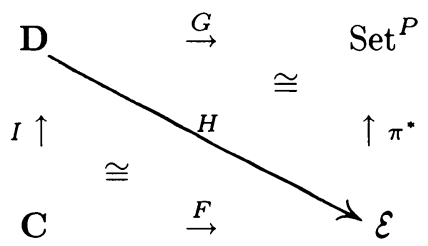

So for any $L \in \operatorname{LEX}(\mathcal{E}$, Set $), L \circ F \cong I^{*}(L H)$, and so $L F \in[\mathcal{K}]$ as required. 3.3

By geometric logic we mean the intuitionistic logic of $=, \wedge, \vee, \exists$ and infinitary disjunction $\bigvee$ (of formulae involving only finitely many free variables); cf. Chapter 2 of $[\mathrm{MR}]$. Specifying a theory $\mathbf{T}$ in this logic amounts to giving a small site $(\mathbf{C}, J)$, i.e., a small category $\mathbf{C} \in \mathbf{L e x}$ equipped with a Grothendieck topology $J$. The category of models of $\mathbf{T}$ in Set, Mod(T), is then the full subcategory of $\operatorname{LEX}(\mathbf{C}$, Set) whose objects are the $J$-continuous functors; this category is equivalent to the category of points of the classifying topos of $\mathbf{T}$, i.e., of the topos of sheaves on the site, $\operatorname{Sh}(\mathbf{C}, J)$. See for example [MR] or Chapter 7 of [TT] for the details of this.

From Theorem 3.3 we can obtain the following result about geometric theories.

3.4 COROLlARY. Suppose that $\mathbf{T}$ is a geometric theory which is specified by a small site $(\mathbf{C}, J)$, where $\mathbf{C}$ has finite limits and the Grothendieck topology $J$ is 
subcanonical (i.e., representable presheaves are sheaves). If $\mathbf{T}$ has enough models in Set (i.e., $\operatorname{Sh}(\mathbf{C}, J)$ has enough points), then

$$
[\operatorname{Mod}(\mathbf{T})]=\mathbf{L E X}(\mathbf{C}, \text { Set }) \text {. }
$$

PROOF. Since $J$ is subcanonical the Yoneda embeding $H: \mathbf{C} \hookrightarrow \operatorname{Set}^{\mathbf{C}^{o p}}$ factors through $\operatorname{Sh}(\mathbf{C}, J) \hookrightarrow \operatorname{Set}^{\mathbf{C}^{\circ \mathbf{P}}}$ and for any $c \in \mathbf{C}$

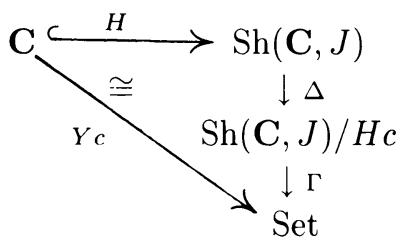

commutes up to isomorphism, where $Y c$ is the representable functor $\mathbf{C}(c,-)$ and $\Delta$ is right adjoint to the forgetful functor $\Sigma: \operatorname{Sh}(\mathbf{C}, J) / H c \rightarrow \operatorname{Sh}(\mathbf{C}, J)$. For $\Gamma \Delta H(-)$ $=\operatorname{Sh}(\mathbf{C}, J) / H c(1, \Delta H(-)) \cong \operatorname{Sh}(\mathbf{C}, J)(\Sigma(1), H(-))=\operatorname{Sh}(\mathbf{C}, J)(H c, H(-)) \cong$ $\mathbf{C}(c,-)$. Applying Theorem 3.3 with $\mathcal{E}=\operatorname{Sh}(\mathbf{C}, J), F=H$ and $L=\Gamma \circ \Delta$, we have

$$
Y c=\Gamma \Delta \circ H \in\left[H_{p} \mid p \in \mathbf{P t}(\mathcal{E})\right] .
$$

But by definition of $\mathcal{E},\left\{H_{p} \mid p \in \mathbf{P t}(\mathcal{E})\right\}=\operatorname{ob} \operatorname{Mod}(\mathbf{T})$. Thus $Y c \in[\operatorname{Mod} \mathbf{T}]$. Now any functor in $\operatorname{LEX}(\mathbf{C}$, Set) can be expressed (canonically) as a filtered colimit of such representable functors. Hence $[\operatorname{Mod} \mathbf{T}]$ is the whole of $\mathbf{L E X}(\mathbf{C}$, Set). $\square 3.4$

3.5 REMARKS. (i) The proof of 3.4 gives no information about the number of times one has to alternate the operations of taking limits and filtered colimits to obtain a particular functor in $\mathbf{L E X}(\mathbf{C}$, Set). We do not know to what extent the maximum number of such alternations is an interesting measure of the complexity of $\mathbf{T}$ as a geometric extension of the finite limit theory $\mathbf{C}$.

(ii) Theorem 3.3 and Corollary 3.4 are best viewed in the context of a general "sheaf representation problem" for geometric theories over finite limit theories, viz:

GIVEN a finite limit theory, i.e., $\mathbf{C} \in \mathbf{L e x}$; a geometric quotient $\mathbf{T}$ of it, i.e., a Grothendieck topology $J$ on $\mathbf{C}$; and a model $F \in \mathbf{L E X}(\mathbf{C}$, Set $)$ of $\mathbf{C}$ in Set.

FIND a Grothendieck topos $\mathcal{E}$ and a sheaf $M$ of $\mathbf{T}$-models over $\mathcal{E}$ (i.e., a $J$ continuous functor in $\operatorname{LEX}(C, \mathcal{E}))$, whose global sections is $F$.

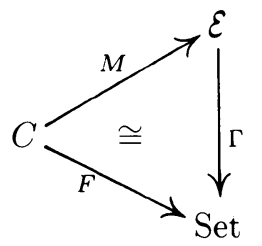

M. Coste $[\mathrm{C}]$ has shown that one can always find such a representation $(\mathcal{E}, M)$ for $F$ provided $J$ is subcanonical and generated by finite covering families (so that $\mathbf{T}$ is a coherent theory). Indeed in this case we can take $(\mathcal{E}, M)$ to be the spectrum of $F$, i.e., the value at $F$ of the left adjoint to the functor

$$
(\mathbf{T} \text {-modelled toposes })^{\text {op }} \rightarrow \mathbf{L E X}(\mathbf{C}, \text { Set })
$$

sending

$$
(\mathcal{E}, M) \mapsto \Gamma \circ M
$$


Returning to the general case, let us remark that recent (unpublished) work of A. Joyal shows that if a representation $(\mathcal{E}, M)$ exists for $F$, then we can always take $\mathcal{E}$ to be localic over Set, i.e., a topos of sheaves on a complete Heyting algebra. For Joyal has shown that for any Grothendieck topos $\mathcal{E}$ there exists a connected, locally connected geometric surjection

$$
q: \operatorname{Sh}(A) \rightarrow \mathcal{E}
$$

with $A$ a complete Heyting algebra (i.e., a locale). This means in particular that $q^{*}$ has a left adjoint $q_{!}: \operatorname{Sh}(A) \rightarrow \mathcal{E}$ satisfying $q_{!}(1) \cong 1$. Hence

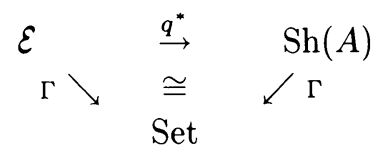

commutes up to isomorphism since

$$
\Gamma q^{*}(-)=\operatorname{Sh}(A)\left(1, q^{*}(-)\right) \cong \mathcal{E}\left(q_{!}(1),-\right) \cong \Gamma(-) .
$$

Thus if $(\mathcal{E}, M)$ is a representation for $F$, so is $\left(\operatorname{Sh}(A), q^{*} M\right)$.

\section{REFERENCES}

[B] J. Benabou, Introduction to bicategories, Lecture Notes in Math., vol. 47, Springer-Verlag, Berlin and New York, 1967, pp. 1-77.

[CK] C. C. Chang and H. J. Keisler, Model theory, North-Holland, Amsterdam, 1973.

[C] M. Coste, Localisation dans les catégories de modeles, Thesis, Univ. Paris Nord, 1977.

[E] D. P. Ellerman, Sheaves of structures and generalized ultra products, Ann. Math. Logic 7 (1974), 163-195.

[GU] P. Gabriel and F. Ulmer, Lokal präsentierbare Kategorien, Lecture Notes in Math., vol. 221, Springer-Verlag, Berlin and New York, 1971.

[GZ] P. Gabriel and M. Zisman, Calculus of fractions and homology theory, Ergebnisse der Mathematik, Band 35, Springer-Verlag, Berlin and New York, 1967.

[TT] P. T. Johnstone, Topos theory, Academic Press, London, 1977.

[FT I] _ Factorization theorems for geometric morphisms. I, Cahiers Topologie et Géom. Différentielle 22 (1981), 3-17.

[KR] A. Kock and G. E. Reyes, Doctrines in categorical logic, Handbook of Mathematical Logic (J. Barwise, ed.), North-Holland, Amsterdam, 1977, pp. 283-313.

[CWM] S. Mac Lane, Categories for the working mathematician, Graduate Texts in Math., vol. 5, Springer-Verlag, Berlin and New York, 1971.

[M] M. Makkai, Remarks on papers by Y. Diers and H. Volger on sheaf representation, Abstracts Amer. Math. Soc. 5 (1984), \#84T-18-76.

[MI _ , Ultraproducts and categorical logic, Methods in Categorical Logic (Ed., C. A. Di Prisco), Lecture Notes in Math., vol. 1130, Springer-Verlag, Berlin and New York, 1985, pp. 222-309.

[MR] M. Makkai and G. E. Reyes, First order categorical logic, Lecture Notes in Math., vol. 611, Springer-Verlag, Berlin and New York, 1977.

[V] H. Volger, Preservation theorems for limits of structures and global sections of sheaves of structures, Math. Z. 166 (1979), 27-53.

[Mu] F. Mouen, Sur la caractérisation sémantique des catégories de structures, Diagrammes 2 (1984).

[AN1] A. Andréka and I. Németi, A general axiomatizability theorem formulated in terms of cone-injective subcategories, Colloq. Math. Soc. J. Bolyai, Esztergom, 1977, pp. 17-35.

[AN2] _ Injectivity in categories to represent all first order formulas. I, Demonstratio Math. 12 (1979), 717-732. 
[GL] R. Guitart and C. Lair, Calcul syntaxique des modèles et calcul des formules internes, Diagrammes 4 (1980), GL1-GL-106.

[NS] I. Németi and I. Sain, Cone implicational subcategories and some Birkhoff type theorems, Colloq. Math. Soc. J. Bolyai, Esztergom, 1977.

[SG A4] Théorie des topos et cohomologie etale des schémas, Eds., M. Artin, A. Grothendieck and J. L. Verdier, Lecture Notes in Math., vol. 269, Springer-Verlag, Berlin and New York, 1972.

Department of Mathematics and Statistics, MCGill University, Montreal, CANADA H3A 2K6 (Current address of M. Makkai)

Department of Mathematical and Physical Sciences, Mathematics DiviSiON, University OF SUSSEX, United KIngdon BN1 9QH (Current address of A. M. Pitts) 